\title{
Fibrilação atrial em cão associada ao quimiodectoma infiltrativo atrial: relato de caso
}

[Atrial fibrillation in dog associated with an infiltrative chemodectoma in the atrial myocardium: a case report]

\author{
G.A.O. Cavalcanti ${ }^{1}$, R.A.L. Muzzi ${ }^{2}$, P.S. Bezerra Júnior ${ }^{2}$, R.B. Nogueira ${ }^{2}$, M.S. Varaschin ${ }^{2}$ \\ ${ }^{1}$ Aluno de pós-graduação - EV-UFMG - Belo Horizonte, MG \\ ${ }^{2}$ Departamento de Medicina Veterinária - UFLA \\ Caixa Postal, 37 \\ 37200-000 - Lavras, MG
}

\begin{abstract}
RESUMO
Relata-se o caso de um cão, sem raça definida, levado ao veterinário por apresentar cansaço fácil e episódios de síncope. Foram identificadas fibrilação atrial e extra-sístoles ventriculares isoladas ao exame eletrocardiográfico. O tratamento antiarrítmico não foi eficaz, e o animal morreu devido a um episódio de síncope. À necropsia, observou-se massa em região de base cardíaca, invadindo a luz do átrio esquerdo. Histologicamente, constatou-se a presença de quimiodectoma. A infiltração miocárdica atrial causou lesões nos miócitos, produzindo circuitos reentrantes e/ou focos ectópicos de despolarização atrial.
\end{abstract}

Palavras-chave: cão, fibrilação atrial, quimiodectoma, infiltração neoplásica

\begin{abstract}
This case reports a mongrel dog referred to a veterinarian due to tiredness and syncope episodes. Atrial fibrillation and ventricular premature complex were observed during electrocardiographic exam. The antiarrhythmic treatment was not effective and the dog died from syncope. At necropsy, a mass invading the internal area of the left atrium was evidenced in the base of the heart. The occurrence of chemodectoma was histologically exhibited. Infiltrations in the atrial myocardium caused alterations in the miocites and produced reentrant circuits and/or atrial depolarization ectopic points.
\end{abstract}

Keywords: dog, atrial fibrillation, chemodectoma, neoplasic infiltration

\section{INTRODUÇÃO}

Os tecidos quimioreceptores estão presentes em vários locais do corpo, porém as neoplasias só se desenvolvem nos quimioreceptores do corpo carotídeo e do aórtico. Esses tecidos são sensíveis a mudanças de $\mathrm{pH}$, temperatura corporal e concentrações de $\mathrm{CO}_{2}$ e de $\mathrm{O}_{2}$ sangüíneos. Eles estimulam os movimentos respiratórios e elevam a pressão arterial e a freqüência cardíaca (Hayes e Sass, 1988; Owen et al., 1996).

Os quimiodectomas de corpo aórtico são cinco vezes mais comuns que os de corpo carotídeo, possuem probabilidade de fazer metástase distante de $12 \%$, sendo freqüente a ocorrência de tumores primários concomitantes, como carcinomas tireoideanos e tumores testiculares (Hayes e Sass, 1988; Owen et al., 1996). Ocorrem mais em machos, com idade superior a oito anos, das raças Boxer e Boston Terrier (Ogilvie, 1989; Capen, 1990), e constituem a principal neoplasia cardíaca canina causadora de doenças pericárdicas (Berg e Wingfield, 1984; Kerstetter et al., 1997).

Humanos, que vivem em locais acima de 2000 metros, apresentam incidência 10 vezes maior desses tumores do que os que vivem no nível do

Recebido em 6 de maio de 2005

Aceito em 17 de abril de 2006

E-mail: guialbuquerque@yahoo.com 


\section{Cavalcanti et al.}

mar, possivelmente devido à hipóxia crônica. A freqüência maior em cães das raças Boxer e Boston Terrier pode ser explicada por predisposição genética, aliada à conformação anatômica de narinas curtas e estenóticas, palato mole alongado e língua grande, dificultando a entrada do ar (Hayes e Sass, 1988; Ogilvie, 1989; Owen et al., 1996). Esses tumores apresentam-se geralmente como massas únicas ou, ocasionalmente, como múltiplos nódulos dentro do saco pericárdico próximo à base cardíaca, podendo infiltrar em pericárdio, epicárdio, miocárdio e parede de grandes vasos (Ogilvie, 1989; Willis et al., 2001).

Os tumores cardíacos podem causar lesões celulares devido ao espaço ocupado e à invasão de tecidos (Robinson e Maxie, 1993). Os que acometem a região atrial podem predispor à taquicardia atrial e/ou fibrilação atrial (Lunney e Ettinger, 1997), por mecanismos de automaticidade anormalmente alterada ou de reentrada miocárdicas (Bigger, 1998).

O mecanismo de automaticidade anormalmente alterada ocorre em miócitos com potencial de membrana em repouso alterados para potencial próximo do limiar de disparo/contração. A alteração do potencial de membrana em repouso ocorre em miócitos isquêmicos e em alterações iônicas (Lunney e Ettinger, 1997; Bigger, 1998). O mecanismo de reentrada pode ocorrer quando um estímulo elétrico encontra área cardíaca com condução elétrica retardada, acarretando reexcitação miocárdica ao sair dessa área e atingir miócitos fora do período refratário (Miller et al., 1996). O mecanismo de reentrada pode ocorrer na região de transição entre o tecido tumoral e o miocárdio normal (Krasuski et al., 2000).

A fibrilação atrial (FA) é caracterizada por contrações atriais desorganizadas que comprometem o débito cardíaco, são detectadas ao exame eletrocardiográfico e apresentam como sinais clínicos: fraqueza, colapso e episódios de síncope (Lunney e Ettinger, 1997; Ramírez et al., 2003).

No exame eletrocardiográfico, a onda $\mathrm{P}$ simboliza a despolarização atrial (Gabay, 2003), e a sua ausência ou ondulações irregulares da linha basal são as alterações eletrocardiográficas observadas em animal com FA (Lunney e Ettinger, 1997; Ramírez et al., 2003).

O exame de escolha que pode sugerir a presença de neoplasias cardíacas é o ecocardiograma (Meng et al., 2002) e, dependendo do caso, o tratamento médico e/ou cirúrgico pode ser feito (Ogilvie, 1989).

Este trabalho teve o objetivo de mostrar que neoplasias infiltrativas podem ser a causa primária de fibrilações atriais que não respondem à terapia.

\section{CASUÍSTICA}

Atendeu-se um cão mestiço Boxer, de 10 anos de idade e $37 \mathrm{~kg}$, com histórico de síncopes esporádicas acompanhadas de cianose e tosse seca há 15 dias.

Ao exame clínico observaram-se crepitações à auscultação pulmonar, arritmias cardíacas, sopro holossistólico de grau V/VI em foco mitral, aórtico e pulmonar e déficit de pulso. O eletrocardiograma mostrou fibrilação atrial (FA) e extra-sístoles ventriculares isoladas (Fig. 1). O tratamento instituído foi furosemida, enalapril, digoxina, descanso e dieta caseira, específica para cães cardiopatas. Após quatro dias, o animal manifestou melhora clínica, mas ao eletrocardiograma as alterações de ritmo ainda estavam presentes. Dessa forma, o atenolol foi adicionado à terapia. Depois de 24 horas de tratamento, o animal manifestou um episódio de síncope e veio a óbito.

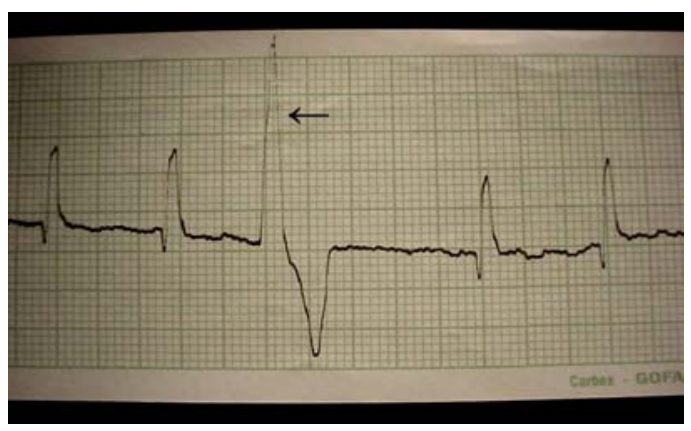

Figura 1. Cão: eletrocardiograma apresentando fibrilação atrial (ondulações na linha de base) e extra-sístole ventricular (seta). Derivação AVF, $50 \mathrm{~mm} / \mathrm{s}$ e sensibilidade $\mathrm{N}$. 
À necropsia, observou-se massa esbranquiçada, com cerca de cinco centímetros de diâmetro, próxima aos vasos da base do coração, circundando parcialmente o tronco pulmonar e completamente o tronco aórtico. Foi também observada infiltração da parede do átrio esquerdo com projeção de cerca de $0,5 \mathrm{~cm}$ de diâmetro na sua luz. O ventrículo esquerdo apresentava hipertrofia concêntrica moderada, e as demais câmaras cardíacas encontravam-se normais (Fig. 2).
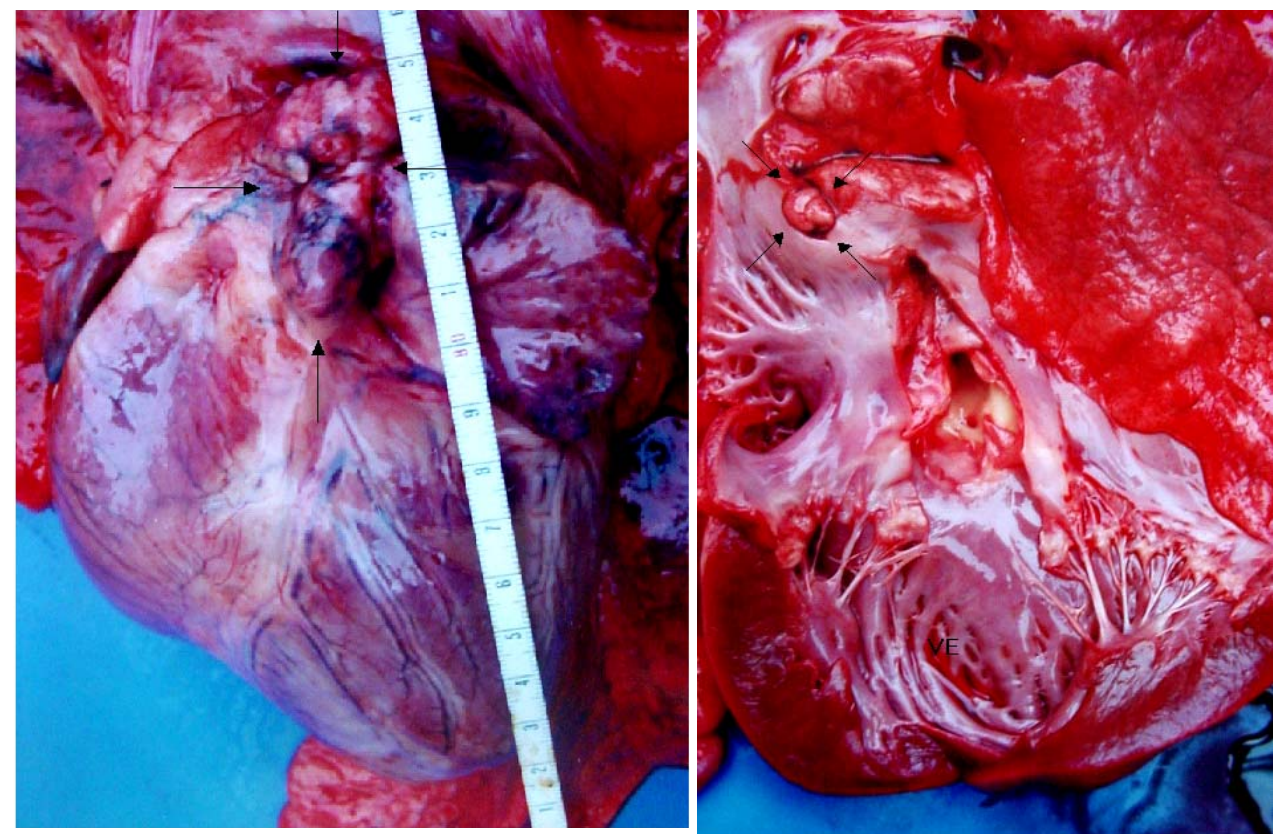

Figura 2. Fotos do coração de cão apresentando massa (quimiodectoma). À esquerda, massa em região dos grandes vasos cardíacos (setas). À direita, massa infiltrando na luz atrial esquerda (setas). VE-ventrículo esquerdo.

Microscopicamente, a massa era constituída por células poliédricas com abundante citoplasma eosinofílico, finamente granular e com anisocitose acentuada. Os núcleos apresentavam pleomorfismo e anisocariose acentuados, cromatina com grumos grosseiros e nucléolos proeminentes, ocasionalmente múltiplos, e com mitoses raras. Células gigantes mononucleares bizarras eram numerosas (Fig. 3). Havia infiltração do miocárdio do átrio esquerdo, que se projetava através do endocárdio em uma área, além da invasão de vasos sangüíneos em alguns campos. Algumas fibras musculares apresentavam necrose nas margens da neoplasia. Estes achados confirmam o diagnóstico de quimiodectoma.

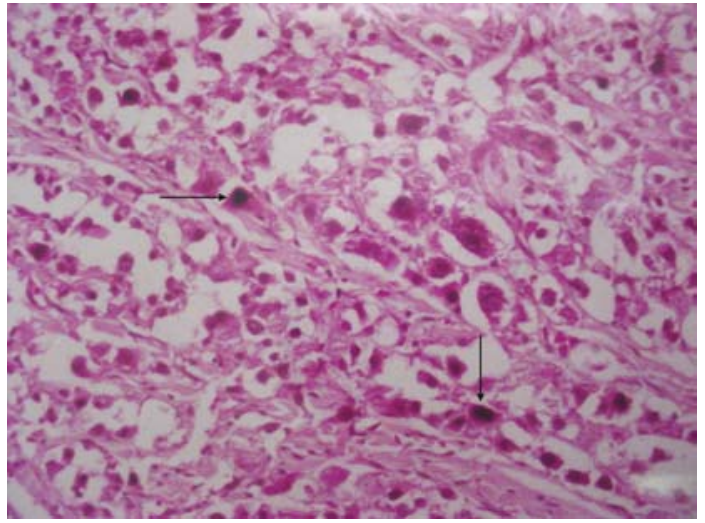

Figura 3. Quimiodectoma de corpo aórtico em um cão. Células arredondadas e poliédricas com citoplasma eosinofílico, finamente granular e anisocitose acentuada. Os núcleos apresentavam pleomorfismo e anisocariose acentuados, cromatina com grumos grosseiros e nucléolos proeminentes. Células gigantes mononucleares bizarras são comuns (seta). 400x, HE. 


\section{DISCUSSÃO}

Em humanos, os quimiodectomas podem secretar catecolaminas (Tekin et al., 2000), mas em cães, por não secretarem hormônios, eles são pouco freqüentes e causam insuficiência diastólica direita devido à efusão e/ou fibrose pericárdicas (Montoya et al., 1995). No presente caso, ocorreu FA.

Lunney e Ettinger (1997) e Ramírez et al. (2003) consideraram a FA como uma das arritmias atriais mais graves e freqüentes na clínica de pequenos animais, sendo geralmente secundária ao aumento atrial. As afecções cardíacas comuns que podem causar aumento atrial são: a cardiomiopatia dilatada, a degeneração mixomatosa crônica de valva mitral e a degeneração mixomatosa crônica de valva tricúspide.

Acredita-se que o sopro sistólico auscultado ocorreu devido à infiltração neoplásica ter acontecido próxima à valva mitral, provocando sua distorção e, conseqüentemente, insuficiência.

A infiltração também acarretou algumas áreas de necrose nas fibras miocárdicas próximas à neoplasia, caracterizadas por condutibilidade diminuída. Sabe-se que os miócitos adjacentes à área tumoral, mesmo histologicamente normais, apresentam alteração de condução elétrica, podendo exibir anormalidades na distribuição da proteína conexina 43 das junções gap, que, segundo Peters e Wit (1998), é a mais abundante das conexinas presentes na membrana celular do miócito.

Os miócitos próximos à área necrosada são de forma irregular e apresentam falhas na distribuição das conexinas, fatores que podem ter levado à condução transversal na região adjacente à necrose (Peters e Wit, 1998). A área necrosada e a área com condução alterada poderiam estar atuando em conjunto na formação de circuitos reentrantes.

Segundo Kittleson e Kienle (1998), a célula miocárdica, quando lesionada ou aumentada de tamanho, pode apresentar modificação no potencial de membrana em repouso, chamada de automaticidade anormalmente alterada.
Os miócitos, com automaticidade anormalmente alterada, têm despolarizações espontâneas, que podem ocasionar complexos prematuros ou taquicardias, dependendo da freqüência das despolarizações (Lunney e Ettinger, 1997; Bigger, 1998). A FA relatada neste caso pode ter sido causada pelo tumor infiltrativo que lesou células do miocárdio.

Os quimiodectomas de corpo aórtico podem causar FA clinicamente intratável, por duas razões: formação de circuitos reentrantes em região adjacente ao tumor e despolarizações ectópicas freqüentes devido a miócitos com automaticidade anormalmente alterada. Os quimiodectomas de corpo aórtico em cães foram, até então, acusados de acarretar apenas efusão e fibrose pericárdicas.

\section{REFERÊNCIAS BIBLIOGRÁFICAS}

BERG, R.J.; WINGFIELD, W.E, Pericardial effusion in the dog: a review of 42 cases. J. Am. Anim. Hosp. Assoc., v.20, p.721-730, 1984.

BIGGER Jr., J.T, Epidemiological and mechanistic studies of atrial fibrillation as a basis for treatment strategies. Circulation, v.98, p.943945, 1998.

CAPEN, C.C. Tumors of the endocrine glands. In: MOULTON, J.E. Tumors in domestic animals. 3.ed. Los Angeles: University of California, 1990. p.553-628.

GABAY, A. Eletrocardiografia. In: BELERENIAN, G.C.; MUCHA, C.J.; CAMACHO, A.A. Afecções cardiovasculares em pequenos animais. 1.ed. São Paulo: Interbook, 2003. p.46-51.

HAYS, H.M.; SASS, B. Chemoreceptor neoplasia: a study of the epidemiological features of 357 canine cases. J. Vet. Med. A, v.35, p.401408, 1988.

KERSTETTER, K.K.; KRAHWINKEL, D.J.; MILLIS, D.L. et al. Pericardiectomy in dogs: 22 cases (1978-1994). J. Am. Vet. Med. Assoc., v.211, p.736-740, 1997.

KITTLESON, M.D.; KIENLE, R.D. Small animal cardiovascular medicine. St. Louis: Mosby, 1998. 603p. 
KRASUSKI, R.A.; HESSELSON, A.B.; LANDOLFO, K.P. et al. Cardiac rhabdomyoma in an adult patient presenting with ventricular arrhythmia. Chest, v.118, p.1217-1221, 2000.

LUNNEY, J.; ETTINGER, S.J. Arritmias cardíacas. In: ETTINGER, S.J.; FELDMAN, E.D. Tratado de medicina interna veterinária. 1.ed. São Paulo: Manole, 1997. p.1338-1382.

MENG, Q.; LAI, H.; LIMA, J. et al. Echocardiographic and pathologic characteristics of primary cardiac tumors: a study of 149 cases. Int. J. Cardiol., v.84, p.69-75, 2002.

MILLER, M.S.; TILLEY, L.P.; DETWEILER, D.K. Eletrofisiologia do Coração. In: SWENSON, M.J.; REECE, W.O. Dukes Fisiologia dos animais domésticos. 11.ed. Rio de Janeiro: Guanabara Koogan, 1996. p.95-132.

MONTOYA, A.J.A.; YNARAJA, R.E.; MARTÍNEZ, A.M.A. et al. Quemodectoma: Una causa de fallo cardíaco congestivo en el perro. cinco casos clínicos. Med. Vet., v.12, p.319-325, 1995.

OGILVIE, G.K. Tumors of the endocrine system. In: WITHROW, S.J.; MACEWEN, E.G. Small animal clinical oncology. 2.ed. Philadelphia: W.B. Saunders, 1989. p.316-346.
OWEN, T.J.; BRUYETTE, D.S.; LAYTON, C.E. Chemodectoma in dogs. Comp. Contin. Educ. Pract. Vet., v.18, p.253-265, 1996.

PETERS, N.S.; WIT, A.L. Myocardial architecture and ventricular arrhythmogenesis. Circulation, v.97, p.1746-1754, 1998.

RAMÍREZ, E.Y.; PALANCA, I.M.; PABLOBLANCO, J.B. et al. Arritmias cardíacas no cão e no gato. In: BELERENIAN, G.C.; MUCHA, C.J.; CAMACHO, A.A. Afecções cardiovasculares em pequenos animais. 1.ed. São Paulo: Interbook, 2003. p.230-259.

ROBINSON, W.F.; MAXIE, M.G. The cardiovascular system. In: JUBB, K.V.F.; KENNEDY, P.C.; PALMER, N. Pathology of domestic animals. 4.ed. California: Academic, 1993. p.1-47.

TEKIN, U.N.; KHAN, I.A.; SINGH, N. et al. A left atrial paraganglioma patient presenting with compressive dysphagia. Can. J. Cardiol., v.16, p.383-385, 2000.

WILLIS, R.; WILLIAMS, A.E.; SCHWARZ, T. et al. Aortic body chemodectoma causing pulmonary oedema in a cat. J. Small Anim. Pract., v.42, p.20-23, 2001. 